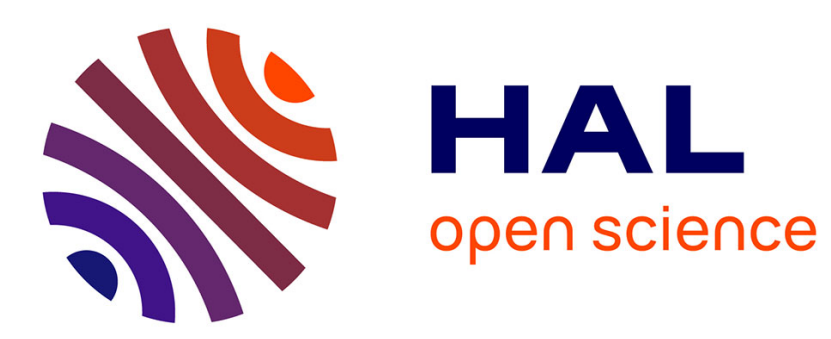

\title{
Precise determination of the ratio $h / m u:$ a way to link microscopic mass to the new kilogram
}

\author{
Pierre Cladé, François Biraben, Lucile Julien, François Nez, Saïda
}

Guellati-Khélifa

\section{- To cite this version:}

Pierre Cladé, François Biraben, Lucile Julien, François Nez, Saïda Guellati-Khélifa. Precise determination of the ratio $\mathrm{h} / \mathrm{m} \mathrm{u}$ : a way to link microscopic mass to the new kilogram. Metrologia, 2016, 53 (5), pp.A75 - A82. 10.1088/0026-1394/53/5/A75 . hal-01394997

\section{HAL Id: hal-01394997 \\ https://hal.sorbonne-universite.fr/hal-01394997}

Submitted on 10 Nov 2016

HAL is a multi-disciplinary open access archive for the deposit and dissemination of scientific research documents, whether they are published or not. The documents may come from teaching and research institutions in France or abroad, or from public or private research centers.
L'archive ouverte pluridisciplinaire HAL, est destinée au dépôt et à la diffusion de documents scientifiques de niveau recherche, publiés ou non, émanant des établissements d'enseignement et de recherche français ou étrangers, des laboratoires publics ou privés. 


\title{
Precise determination of the ratio $h / m_{\mathrm{u}}$ : a way to link microscopic mass to the new kilogram
}

\author{
Pierre Cladé $^{1}$, François Biraben ${ }^{1}$, Lucile Julien ${ }^{1}$, François Nez ${ }^{1}$ \\ and Saïda Guellati-Khelifa ${ }^{1,2}$ \\ ${ }^{1}$ Laboratoire Kastler Brossel, UPMC-Sorbonne Universités, CNRS, ENS-PSL \\ Research University, Collège de France, 4 place Jussieu, 75005 Paris, France \\ ${ }^{2}$ Conservatoire National des Arts et Métiers, 292 rue Saint Martin, 75003 Paris, \\ France \\ E-mail: saida.guellati@lkb.upmc.fr
}

\begin{abstract}
.
The ratio $h / m_{\mathrm{u}}$ between the Planck constant and the unified atomic mass constant should have a special status in the framework of the future International System of Units. Currently (before the redefinition), this ratio allows to compare determinations of $h$ (watt balance) and determinations of $m_{\mathrm{u}}$ (XRCD method). In the future SI, as the Planck constant $h$ will be fixed, the ratio $h / m_{\mathrm{u}}$ will ensure the realization of the new kilogram (quantum kilogram) at the atomic scale. Furthermore as the Avogadro constant will be fixed, the carbon molar mass $\mathrm{M}\left({ }^{12} \mathrm{C}\right)$, which will no longer be equal to $12 \mathrm{~g} \cdot \mathrm{mol}^{-1}$, will be determined from $m_{\mathrm{u}}$. This ratio is also a key data for the realization of the kilogram at the macroscopic scale using the XRCD method.

In this paper we present the state of the art on experiments that provide the most precise value of the ratio $h / m_{\mathrm{u}}$. We focus on the one based on the measurement of the atomic recoil due to the photon momentum.
\end{abstract}

The kilogram is the last remaining base unit of the International System of Units which is still defined by a material artifact, the International Prototype of the Kilogram (IPK). Over the past 25 years, an international effort has been underway to change this definition and base it on a fundamental principle. Two different new definitions have been foreseen in the past : a first definition based on atomic mass (for example by fixing the value of the unified atomic mass constant $\left.m_{\mathrm{u}}=m\left({ }^{12} \mathrm{C}\right) / 12 .^{\ddagger}\right)$ and a second one based on the Planck constant $h$ [1]. Those two definitions are related to two experimental methods: the silicon X-ray crystal density method (XRCD) $[2,3,4]$ and the watt balance experiment $[5,6,7,8]$ which link to the kilogram respectively the atomic mass constant and the Planck constant (see Figure 1).

$\ddagger m_{\mathrm{u}}$ is the mass of the unified atomic mass unit $\mathrm{u}$ (also called the dalton, Da). In this paper, we choose not to use this unit which is not a SI unit, but instead we use the physical constant $m_{\mathrm{u}}$. Note also, that currently (before the redefinition), the product between $m_{\mathrm{u}}$ and the Avogadro constant $N_{\mathrm{A}}$ is fixed by the relation $m_{\mathrm{u}} N_{\mathrm{A}}=1 \mathrm{~g} \cdot \mathrm{mol}^{-1}$. We choose not to mention the Avogadro constant in the paper because its definition will change with the new SI. 


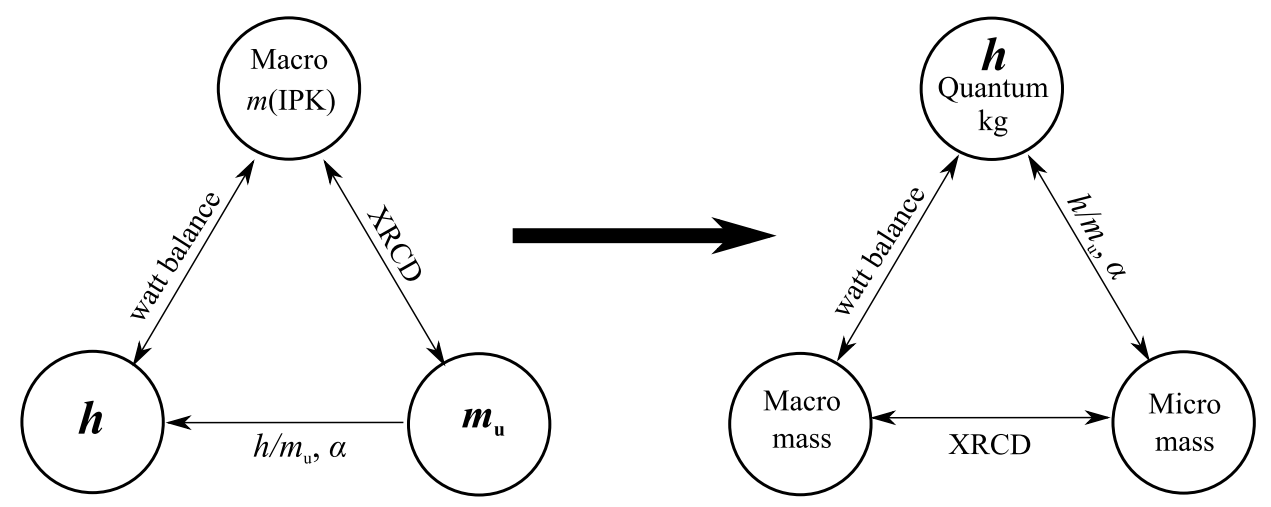

Figure 1. In the current SI, the ratio $h / m_{\mathrm{u}}$ provides a direct comparison between the watt balance experiment and the XRCD experiment. In the future SI this ratio should be consistent with the draft of the Mise en Pratique of the new kilogram. It would allow the realization of the kilogram at the atomic scale and at the macroscopic scale using the XRCD method.

The choice of the CGPM 2014 for the new definition of the kilogram is to fix the value of the Planck constant[9]. This choice highlights the watt balance method. However, the XRCD method is still relevant and the two methods will be considered in the mise en pratique of the kilogram. Indeed, using the ratio $h / m_{\mathrm{u}}$ which is measured precisely, one can link the Planck constant to the mass $m$ of the silicon sphere used in the XRCD method with the formula:

$$
m=h \frac{8 V}{a_{0}^{3}} \frac{m_{\mathrm{u}}}{h} A_{\mathrm{r}}(\mathrm{Si})
$$

where $V$ is the volume of the sphere, $a_{0}$ is the lattice constant and $A_{\mathrm{r}}(\mathrm{Si})$ is the mean relative atomic mass in the silicon crystal. It can be calculated using the well-known values of $A_{\mathrm{r}}\left({ }^{{ }^{x} \mathrm{Si}}\right)=m_{\mathrm{\times} \mathrm{Si}} / m_{\mathrm{u}}$, where $m_{\mathrm{\times Si}}$ is the mass of the silicon isotope $\mathrm{x}$.

Currently, before the redefinition, this equation allows to get a determination of $h$ from the XRCD method as recommended by the CCM [10]. After the redefinition, it will be part of the realization of the kilogram using the XRCD method [11]. In this equation, we have introduced the ratio $h / m_{\mathrm{u}}$. The purpose of this article is to describe how it is precisely determined.

The CODATA (Committee on Data for Science and Technology)-TGFC (Task Group on Fundamental Constants) will be in charge of the determination of the numerical values of the constants that will be used in the new definition [9]. The method of the CODATA consists in extracting the value of a constant using a least square adjustment based on all relevant experiments. While this method combines all measurements together, it is still possible to extract an individual path to obtain a value of $h / m_{\mathrm{u}}$. We will present in this paper the two main ways for a measurement of $h / m_{\mathrm{u}}$ : a direct measurement obtained from a measurement of $h / m_{\mathrm{X}}$ where $\mathrm{X}$ is an atomic species $\left(m_{\mathrm{X}}\right.$ its mass), and an indirect measurement using the Rydberg constant $R_{\infty}$ and the fine structure constant $\alpha$. 
The direct method relies on the measurement of the Doppler shift induced by the recoil of an atom absorbing a photon. This shift is given by

$$
\nu_{D}=\frac{1}{\lambda_{R} \lambda_{B}} \frac{h}{m_{\mathrm{X}}}
$$

where $\lambda_{R}$ is the wavelength of the laser used for measuring the Doppler effect and $\lambda_{B}$ the wavelength of the photon inducing the recoil. With the new definition, this atomic recoil measurement will be a direct method for measuring the mass of $\mathrm{X}$ [12]. It also allows to determine $h / m_{\mathrm{u}}=h / m_{\mathrm{X}} \times A_{\mathrm{r}}(\mathrm{X})$, because the relative atomic mass $A_{\mathrm{r}}(\mathrm{X})=m_{\mathrm{X}} / m_{\mathrm{u}}$ of an atomic entity $\mathrm{X}$ is measured precisely [13].

The second method, also based on atomic physics, relies on the measurement of the Rydberg constant $R_{\infty}$ using hydrogen spectroscopy:

$$
R_{\infty}=\frac{m_{\mathrm{e}} \alpha^{2} c}{2 h}
$$

where $\alpha$ is the fine-structure constant and $m_{\mathrm{e}}$ is the electron mass.

Those two experiments are the most precise experiments used to get the ratio between $h$ and a microscopic mass (atomic mass or electron mass). Currently they allow for an uncertainty on the ratio $h / m_{\mathrm{u}}$ of about $1.3 \times 10^{-9}$ for the direct method and $5.0 \times 10^{-10}$ for the measurement based on the fine structure constant. Nowadays, before the redefinition, the role of the ratio $h / m_{\mathrm{u}}$ is to extract a value of $h$ using the XRCD method. The CCM recommends measurements of $h$ with independent methods in order to validate the new SI. The uncertainty required to validate a measurement is $2 \times 10^{-8}$ [10], therefore, the uncertainty on the determination of $h / m_{\mathrm{u}}$ is not a limiting factor. This can be seen in the correlation factor between $m_{\mathrm{u}}$ and $h$ in the CODATA 2014 which is 0.9993.

Finally, we want to emphasize the importance of this ratio in the framework of the new SI. In quantum mechanics, the inertial mass $m$ is always combined with the Planck constant $h$. Any measurement of a quantity directly related to a mass, is a measurement of $h / m$ and therefore, within the future definition, is a direct measurement of a mass at the atomic scale. Since in the SI, the speed of light $c$ is fixed, this can be also interpreted

as an indirect determination of the Compton frequency $h / m c^{2}$ of the particle. This physical concept has been proposed for the definition of the kilogram [14].

The next two sections are devoted to the presentation of the state of the art as well as the perspective for the two most promising determinations of the ratio $h / m_{\mathrm{u}}$.

\section{Determination of the ratio $h / m_{\mathrm{u}}$ from the fine structure constant $\alpha$}

An accurate determination of $h / m_{u}$ can be derived from the values of fundamental constants using the relationship:

$$
\frac{h}{m_{\mathrm{u}}}=\frac{\alpha^{2} c A_{\mathrm{r}}(\mathrm{e})}{2 R_{\infty}}
$$

in which $A_{\mathrm{r}}(\mathrm{e})=m_{\mathrm{e}} / m_{\mathrm{u}}$ is the relative atomic mass of the electron, $R_{\infty}$ is the Rydberg constant and $\alpha$ is the fine structure constant. 
We will denote $\frac{h}{m_{\mathrm{u}}}(\alpha)$ a value of $h / m_{\mathrm{u}}$ obtained using this equation. Currently, this value is limited by the measurement of the fine structure constant.

\subsection{Determination of $A_{\mathrm{r}}(\mathrm{e})$}

The determination of $A_{\mathrm{r}}(\mathrm{e})$ is the measurement of the ratio between two masses: the mass of the electron $m_{\mathrm{e}}$ and the unified atomic mass constant $m_{\mathrm{u}}$. Until recently, the only method available for the measurement of mass ratio was based on the measurement of the cyclotron frequency $\nu_{c}$ in a Penning trap. The ratio of two measurements of $\nu_{c}$ gives the ratio of the masses. This method is widely used for the measurement of relative atomic masses. Depending on the atom, this quantity is known with an uncertainty of typically $10^{-8}$ to $10^{-11}$. The Atomic Mass Data Center (AMDC) carries out from timeto-time a comprehensive atomic mass evaluation (AME) and publishes a list of many hundreds of relative atomic-mass values. Their latest available paper was published in $2012[13,15,16]$. We want to emphasize that the atomic masses are linked to the SI at the level of $10^{-8}$ while in the new SI they will be linked to the kilogram at the level of $10^{-9}$.

The best determination of $A_{\mathrm{r}}(\mathrm{e})$ is realized in a special Penning trap by a German collaboration with a relative uncertainty of $2.9 \times 10^{-11}$ [17]. The atomic mass of the electron is determined combining a precise measurement of the Larmor $\left(\nu_{L}\right)$ to cyclotron $\left(\nu_{c}\right)$ frequency ratio on a single hydrogen-like ion $\left({ }^{12} \mathrm{C}^{5+}\right)$ with a value of the $g$-factor in the following equation:

$$
m_{\mathrm{e}}=\frac{g}{2} \frac{e}{q} \frac{\nu_{c}}{\nu_{L}} m_{\text {ion }}
$$

The hydrogen-like carbon ion of mass $m_{\text {ion }}$ and charge $q$ is the defining particle for the atomic mass (apart from the mass and binding energies of the missing electrons, which are sufficiently well known). Advances in quantum electrodynamics (QED) in the past few years allow to calculate the $g$-factor with higher precision [18]. This, added to the impressive progress in the control of ions' motion in a Penning trap, led recently to the determination of $A_{\mathrm{r}}(\mathrm{e})$ with a precision that surpasses the current literature value of the CODATA 2010 by a factor of 13 [17].

\subsection{Determination of the Rydberg constant $R_{\infty}$}

The Rydberg constant is the third best known fundamental constant. Its value is provided by high resolution spectroscopy of hydrogen and deuterium atoms ([19] and ref herein). The main contributions are the ones obtained in Garching in the group of T.W. Hänsch on the 1S-2S transition [20] and the ones obtained in Paris, in the group of $\mathrm{F}$. Biraben on the $2 \mathrm{~S}-\mathrm{nS} / \mathrm{nD}$ transitions [21]. The latest value of $R_{\infty}$ is given by the 2014 adjustment of CODATA with a relative uncertainty of $5.9 \times 10^{-12}$ [22] However, this accurate value is questioned by the recent determination of the proton radius from muonic hydrogen spectroscopy [23, 24] which is in contradiction with the radius obtained from hydrogen spectroscopy. The problem can be solve by shifting the 
value of the Rydberg constant by 5.8 times its current uncertainty [23, 24]. Fortunately, this value of $R_{\infty}$ shifts insignificantly the determination of $h / m_{\mathrm{u}}(\alpha)$.

\subsection{Determination of the fine structure constant $\alpha$}

Since 1987, the best determination of $\alpha$ arises from the measurement of the anomalous moment of the electron $a_{\mathrm{e}}$ in a Penning trap combined with QED calculations of $a_{\mathrm{e}}$.

The value of $a_{\mathrm{e}}$ is determined by $a_{\mathrm{e}}=\nu_{a} / \nu_{c}$ where $\nu_{a}=\nu_{s}-\nu_{c}, \nu_{c}=e B / 2 \pi m_{\mathrm{e}}$ is the cyclotron frequency, and $\nu_{s}=g_{\mathrm{e}} \mu_{B} B / h$ is the spin-flip (or precession) frequency. In this experiment, $\nu_{a}$ and $\nu_{c}$ are measured in the same magnetic flux density $B$ of $\sim 5 \mathrm{~T}$. Twenty years of developments of new methods in the group of G. Gabrielse, at Harvard University, have been necessary to realize the best experimental determination of $a_{\mathrm{e}}[25,26]$ to date with a relative uncertainty of $0.28 \times 10^{-12}$. A new trap is under development at Harvard to improve the accuracy of $a_{\mathrm{e}}$ [27].

The most precise determination of $\alpha$ is obtained from the above experiment combined with the latest challenging QED calculations (tenth order term !) done in the group of T. Kinoshita and M. Nio [28, 29]. It has a relative uncertainty of $2.5 \times 10^{-10}$.

This determination has varied in the past because of re-evaluations of QED calculations (see [19] and ref. therein) which have shifted the value of $\alpha$ whereas the experimental value of $a_{\mathrm{e}}$ has been measured twice in Harvard with an excellent agreement $[25,26]$. However, in the near future this situation should be enlightened with a competitive determination of $\alpha$ from bound electron g-factor experiments combined with a weighted difference of the $g$-factor of $\mathrm{H}$ - and Li-like ions of the same elements [30].

Using the value of the fine structure constant from $a_{\mathrm{e}}[29]$, the current value of $h / m_{\mathrm{u}}(\alpha)$ is then:

$$
\frac{h}{m_{\mathrm{u}}}(\alpha)=3.9903127099(20) 10^{-7} \mathrm{~m}^{2} \cdot \mathrm{s}^{-1}
$$

in which the main component of the uncertainty is due to $\alpha$.

\section{Determination of the ratio $h / m_{\mathrm{u}}$ from the photon recoil measurement.}

A direct determination of the ratio $h / m_{\mathrm{X}}$ between the Planck constant and an atomic mass can be obtained from the measurement of the recoil velocity $v_{\mathrm{r}}=\hbar k / m_{\mathrm{X}}$ of an atom when it absorbs a photon of momentum $\hbar k$ ( $k$ is the wave vector). Since the relative atomic mass is known with a high precision this leads to the ratio $h / m_{\mathrm{u}}$.

The recoil measurement is based on a Ramsey-Bordé atom interferometer according to the pioneering idea proposed by S. Chu in 1993 [31]. Such an atom interferometer uses two pairs of $\pi / 2$ light-pulses (see Figure 2). Each light pulse induces a two-photon Raman transition between the two hyperfine levels of the ground state. As the Raman transition is performed by two counter-propagating laser beams, an atom absorbs a photon from one beam and re-emits a stimulated photon towards the other beam. Then 
it imparts to the atom $2 \times v_{\mathrm{r}}$. Such transitions are used to separate and recombine the atomic wave packets. Because the Raman transitions are Doppler sensitive, the RamseyBordé atom interferometer measures the velocity change that occurs between the first and the second pair of light pulses. The velocity distribution of atoms transferred after the first two $\pi / 2$-pulses follows a Ramsey fringe pattern. The fringe interval $\Delta v$ of this distribution is equal to:

$$
\Delta v=\frac{h}{m} \times \frac{1}{\Delta x}
$$

where $\Delta x$ is the distance between the two arms of the interferometer. The second pair of $\pi / 2$ pulses closes the interferometer by transferring the atoms to the initial state with a probability that follows a Ramsey fringe pattern. One can scan the fringes by changing the frequency of the Raman lasers during the second pair of light pulses. The frequency of the central fringe corresponds to a frequency shift that compensates any Doppler effect endured by the atoms within the interferometer.

In the initial scheme [32], the frequency Doppler shift was due to the recoil velocity induced by the Raman transition. In order to improve the sensitivity to the recoil velocity, S. Chu proposed to increase the number of photon momenta transferred to the atoms in the middle of the atom interferometer [31]. Nowadays the most promising approach is the one that uses Bloch oscillations in an accelerated lattice [33, 34] instead of the starting approach based on a sequence of Raman pulses [35]. Bloch oscillations (BO) occur when atoms are placed in an standing wave which is accelerated by linearly sweeping the relative frequency of two counter-propagating laser beams [36, 37]. The physics behind the Bloch oscillation phenomenon is detailed in [38]. A simple picture to understand the coherent acceleration induced by the BO technique is to consider that the atom undergo a succession of Raman transitions in which they begins and ends in the same energy level. The internal state is unchanged while the atomic velocity increases by $2 \times v_{\mathrm{r}}$ (Bragg diffraction). The Doppler shift due to this velocity change is periodically compensated by the frequency sweep of the Bloch laser and the atom is accelerated. The velocity of atoms increases by $2 \times v_{\mathrm{r}}$ per BO: for 87 -rubidium it corresponds to a $30 \mathrm{kHz}$ Doppler shift. The number of $\mathrm{BO}$ is set by the frequency sweep range. Note that $\mathrm{BO}$ is a very efficient process, for which we demonstrate that more than $90 \%$ of the atoms get a total velocity variation of $1000 \times v_{\mathrm{r}}(500 \mathrm{BO})$.

\subsection{Experimental set-up}

Figures 3 and 4 show an overview of our experimental setup in Paris [33]. We use a source of about $2 \times 10^{8}$ cold rubidium atoms in the $F=2$ hyperfine level at a temperature of $4 \mu \mathrm{K}$. This atomic source is produced in a three-dimensional magneto-optical trap loaded by a slow beam (about $10^{9}$ atoms/s at a velocity of $20 \mathrm{~m} / \mathrm{s}$ ) coming from a two-dimensional magneto-optical trap (2D-MOT).

A key point in this experiment is to stabilize and control the frequency and phase difference between the two Raman lasers used for the interferometer. The lasers are 


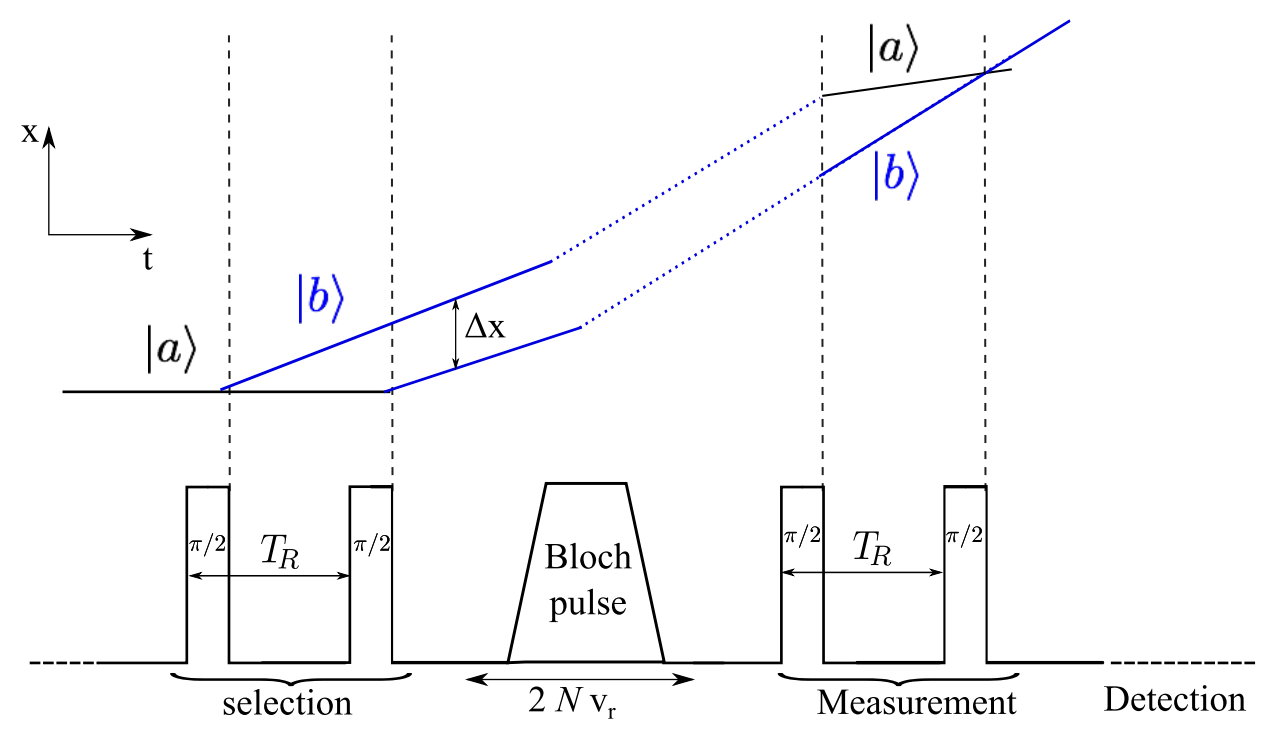

Figure 2. Top figure: description of the semi-classical trajectories of atoms in the atom interferometer. Only the trajectories that play a role in the interferometer are displayed. Bottom figure: timing sequence of the light pulses used for the atom interferometer and Bloch oscillations.

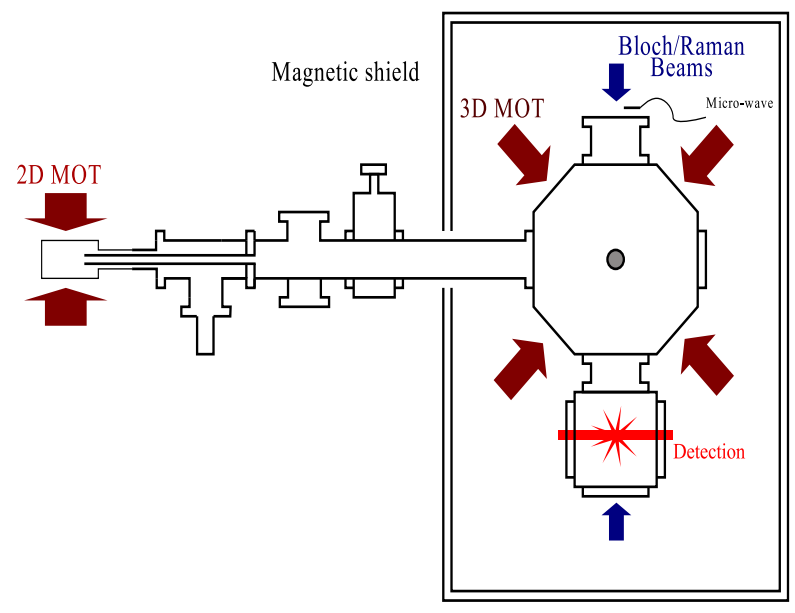

Figure 3. Scheme of the vacuum chamber. The cold atomic cloud is produced in a $3 \mathrm{D}-\mathrm{MOT}$ loaded by a slow atomic beam (from a 2D-MOT). The Raman and the Bloch beams are in vertical geometry. The populations in each hyperfine level are detected by fluorescence at $15 \mathrm{~cm}$ below the 3D-MOT using a time-of-flight technique.

phase-locked using a synthesized frequency referenced to a caesium atomic clock. As shown in Figure 4, the synthesized frequency results from the mixing of a fixed frequency $(6.834 \mathrm{GHz})$, a frequency ramp to compensate the fall of atoms in the gravity field (about $25 \mathrm{MHz} / \mathrm{s}$ ) and the probe frequency. The probe frequency is switched between $\delta_{\text {sel }}$ and $\delta_{\text {meas }}$ using two independent synthesizers, where $\delta_{\text {sel }}$ and $\delta_{\text {meas }}$ are the frequency differences between the two Raman beams respectively during the first and the second pair of $\pi / 2$ pulses. To implement Bloch oscillations, we need to generate a moving 


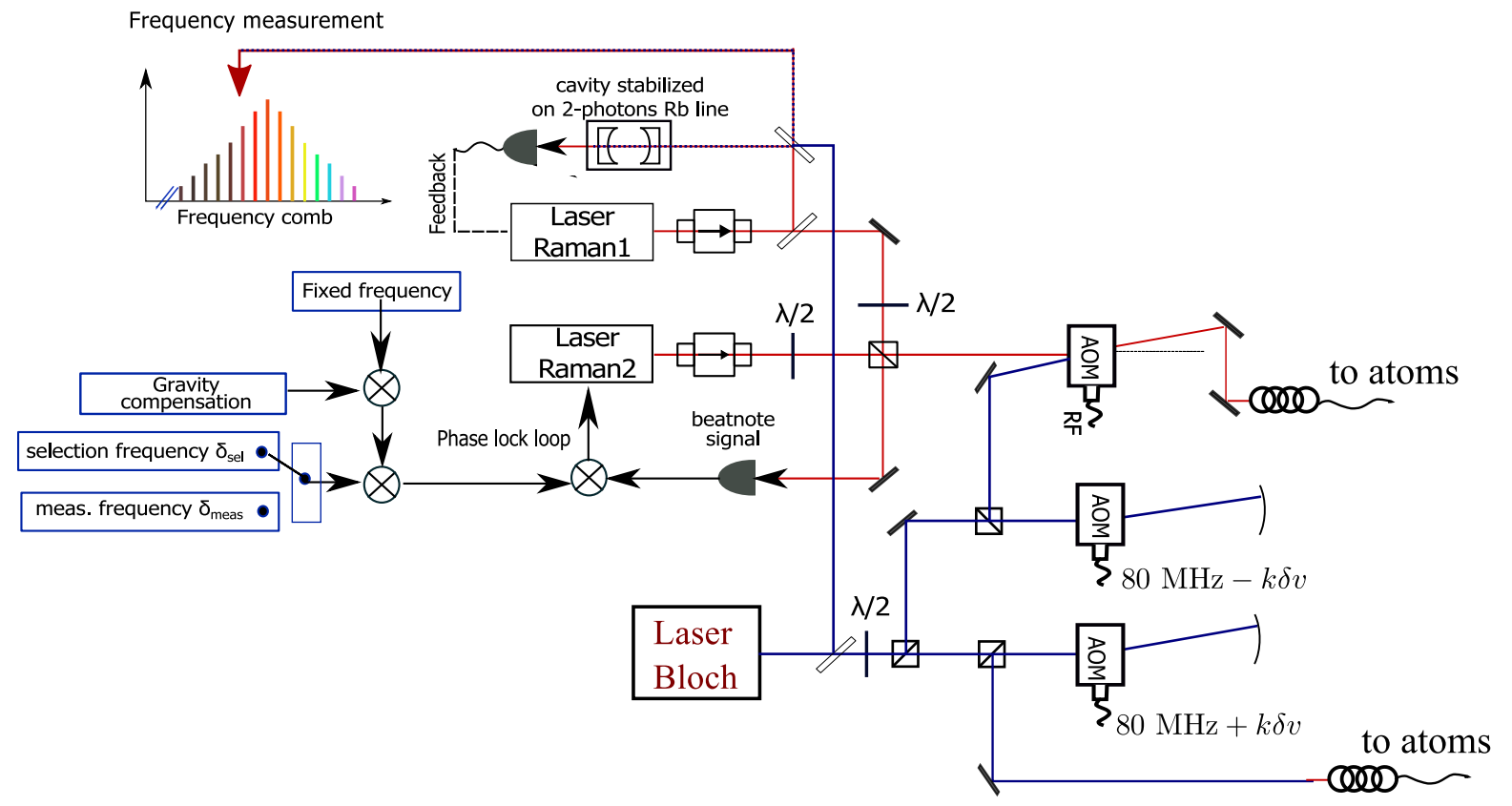

Figure 4. Optical setup of the Raman beam, used to perform the atomic interferometer, and the Bloch beams. The Raman lasers are phase locked. The frequencies of one Raman and of the Bloch lasers are stabilized on an ultra-stable cavity and measured with a frequency comb.

optical lattice with a controlled velocity. This requires a high power laser source $(2.5 \mathrm{~W}$ Ti:sapphire laser). The Bloch beam is split into two paths, each of which passes through an acousto-optic modulator (AOM) to adjust the frequency offset (velocity of the lattice) and amplitude. The depth of the generated optical lattice is $45 E_{r}$ ( $E_{r}$ is the recoil energy). In order to get a value of the ratio $h / m$, we need to precisely measure the wavelength of the Raman and Bloch lasers (equation 2). The frequencies of one Raman laser and the Bloch laser are stabilized onto the same ultra-stable Zerodur Fabry-Perot cavity, itself stabilized on the $5 S_{1 / 2}(F=3) \longmapsto 5 D_{5 / 2}(F=5)$ two-photon transition

of 85-rubidium (for the short term stability). On the long term, these frequencies are precisely measured by using a femtosecond comb referenced to the caesium clock. As the measurement of the ratio $h / m_{\mathrm{Rb}}$ is performed in terms of frequency, it is thus directly connected to the caesium standard (Figure 4).

\subsection{Results and recent improvement}

In this section, we will summarize the results obtained during the 2010 measurement campaign in Paris [33].

A typical fringe pattern is shown in Figure 5. It is recorded with 100 points during $1 \mathrm{~min}$. The central fringe is determined with an uncertainty of $0.14 \mathrm{~Hz}$ corresponding to a relative uncertainty of $10^{-8}$ on the Doppler shift $\left(\delta_{\text {sel }}-\delta_{\text {meas }}\right)$ induced by 500 BO.

To prevent systematic errors and gravity, a value of $h / m_{\mathrm{Rb}}$ is determined from four 


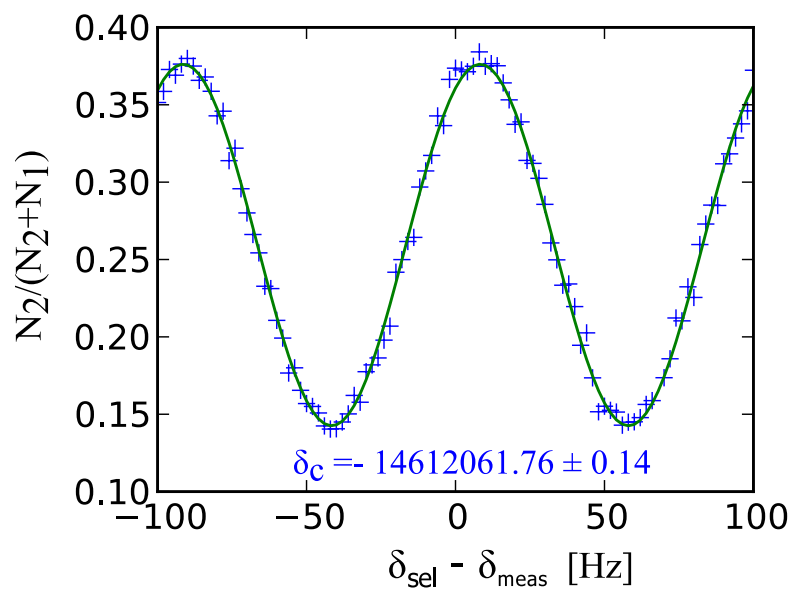

Figure 5. The quantity $N_{2} /\left(N_{2}+N_{1}\right)$ versus the frequency difference between the two pairs of $\pi / 2$ pulses, where $N_{2}$ and $N_{1}$ represent respectively the populations in hyperfine levels $F=1$ and $F=2$. The spectrum is recorded with 100 points during 1 min. The measured position of the central fringe is indicated under the spectrum.

spectra. Following the experimental protocol, two spectra are obtained by inverting the direction of the Raman beams in order to eliminate the parasitic level shifts due to the Zeeman effect and the light shifts. For each beam direction we accelerate the atoms alternatively upward and downward to get rid of the change in velocity due to the free fall of atoms in the gravity field. The experimental protocol and the corresponding timing sequence are detailed in [33, 39].

A set of 170 measurements of the ratio $h / m_{\mathrm{Rb}}$ obtained during 15 hours of integration time leads to a standard deviation of the mean value of $4.4 \times 10^{-10}$.

The systematic effects, which are the dominant part of the final uncertainty, are detailed in [33, 39]. The effects taken into account are: laser frequencies, beam alignment, second order Zeeman effect, gravity gradient, Gouy phase and wave front curvature. The resulting values of the ratios $h / m_{\mathrm{Rb}}$ and $h / m_{\mathrm{u}}$ are:

$$
\begin{aligned}
\frac{h}{m_{\mathrm{Rb}}} & =4.5913592729(57) \times 10^{-9} \mathrm{~m}^{2} \cdot \mathrm{s}^{-1} \\
\frac{h}{m_{\mathrm{u}}} & =3.9903127193(51) \times 10^{-7} \mathrm{~m}^{2} \cdot \mathrm{s}^{-1}
\end{aligned}
$$

The main systematic effect in the Paris experiment comes from the Gaussian profile of the laser beams. The atoms experience an effective wavevector determined by the gradient of the laser phase along the propagation axis z:

$$
k_{\mathrm{eff}}=\frac{d \phi}{d z}=k-\frac{2}{k}\left[\frac{1}{w^{2}}-\frac{r^{2}}{w^{4}}+\frac{k^{2} r^{2}}{4 R^{2}}\right]
$$

where $r$ is the radius of the atomic cloud, $w$ the waist of the laser and $R$, the curvature radius. This formula includes both contributions of the Gouy phase and the wave front curvature. The geometrical parameters of the laser beams have been carefully measured 
with a Shack-Hartmann wave-front analyzer. This effect is common to all kinds of measurements based on atom interferometry and also to any distance measured with an optical interferometer - for example in the watt balance or the XRCD method.

The best way to reduce this effect is to increase the waist of the laser beams and to reduce the size of the atomic cloud. Recently we developed a high power laser source for Bloch oscillations and atom interferometry. They are based on the frequency doubling of an amplified seed laser at $1560 \mathrm{~nm}$ in a PPLN (periodically poled lithium niobate) crystal [40]. The available laser powers will allow to increase the beam waists at least by a factor of two.

Furthermore, a new experimental setup is under construction. We aim at using a smaller and colder atomic source based on evaporative cooling. With a narrow size atomic source the Gouy phase and wave front curvature systematic effects will be reduced.

The next step is to realize an atom interferometer with a large momentum beamsplitter based on Bloch oscillations. Indeed, increasing the recoil transferred by the beamsplitter will increase the distance between the two arms of the interferometer and therefore its sensitivity (equation 1). In order to implement such a beamsplitter, an atomic source with narrow spatial and momentum spread is required to reduce the phase fluctuations due to light shifts during Bloch oscillations [41, 42] which reduce the fringe contrast. However, the drawback of the high-density atomic source is the atomatom interactions which give rise to density-dependent mean-field shifts in the atomic interferometer. A precise evaluation of this effect is currently under investigation in our group [43].

The S. Chu pioneering experiment which provided a preliminary measurement of the ratio $h / m_{\mathrm{Cs}}$ in 2002 with a relative uncertainty of $1.4 \times 10^{-8}$ [35], is now under the supervision of H. Müller at UC Berkeley. This group obtained many impressive results toward a new determination of this ratio [44] and published a value with an uncertainty of $4 \times 10^{-9}$ in 2013 [45]. They recently significantly enhanced the sensitivity of the atom interferometer thanks to a combination of Bragg diffraction and Bloch oscillations. They obtained a promising resolution of $5 \times 10^{-10}$ on the caesium recoil frequency measurement [34]. Unfortunately, the Bragg diffraction technique used to implement the atomic beamsplitter causes a diffraction phase, which induces an embarrassing systematic effect in the atom interferometer. They recently managed to control this effect [34] and they will probably provide a new determination of $h / m_{\mathrm{Cs}}$ in the near future.

\section{Discussion}

The different determinations of the ratio $h / m_{\mathrm{u}}$ are shown in Figure 6 . The values labelled (Cs recoil) and ( $\mathrm{Rb}$ recoil) are deduced from the atomic recoil measurements performed respectively with caesium atoms in H. Müller's group at UC Berkeley [45] and rubidium atoms at Laboratoire Kastler Brossel in Paris. Currently the relative standard uncertainties for $A_{\mathrm{r}}\left({ }^{133} \mathrm{Cs}\right)$ and $A_{\mathrm{r}}\left({ }^{87} \mathrm{Rb}\right)$ are $6.5 \times 10^{-11}$ and $7.5 \times 10^{-11}$, respectively 


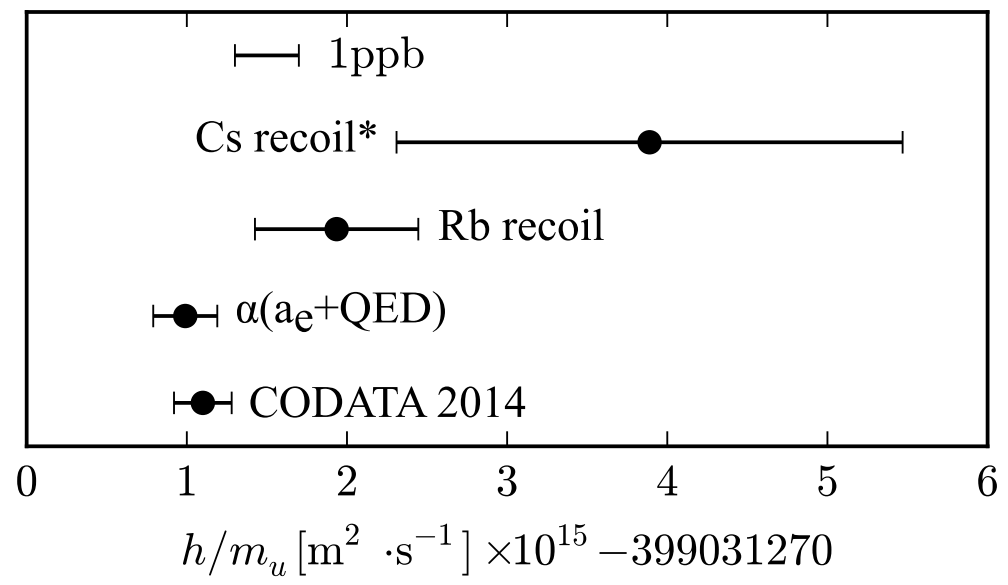

Figure 6. Determinations of the ratio $h / m_{\mathrm{u}}$ issued from the recoil measurement (cesium and rubidium) and from the fine structure constant value deduced from the measurement of the electron moment anomaly and QED calculations.

(*) This value is not included in the last least square adjustment of the CODATA for the determination of the recommended values of fundamental constants. Some small corrections have recently been identified, that were not yet taken into account for its determination [46].

and the smallest relative uncertainty for the direct atom-interferometry measurements of $h / m\left({ }^{133} \mathrm{Cs}\right)$ and $h / m\left({ }^{87} \mathrm{Rb}\right)$ are $1.5 \times 10^{-8}$ and $1.2 \times 10^{-9}$, respectively. It should be noted that the Berkeley value is not included in the last least square adjustment of the CODATA for the determination of the recommended values of fundamental constants. Some small corrections have recently been identified, that were not yet taken into account for the determination of the caesium recoil value ([46] and ref herein).

The value $h / m_{\mathrm{u}}$ labelled $\alpha\left(a_{e}+Q E D\right)$ is deduced from equation 4 using the recent determination of the relative electron mass [17], the CODATA 2014 recommended value of the Rydberg constant and the value of the fine structure constant $\alpha$ obtained from the measurement of electron anomaly, made by the group of Gabrielse at Harvard university [26] and the recent QED calculations reported in the last publication of Nio and Kinoshita's group at Nishina Center in Japan [29]. We have also included in Figure 6 the CODATA 2014 recommended value:

$$
\frac{h}{m_{\mathrm{u}}}(\text { CODATA2014 })=3.9903127110(18) \times 10^{-7} \mathrm{~m}^{2} \cdot \mathrm{s}^{-1}\left[4.5 \times 10^{-10}\right]
$$

In comparison with the situation in the CODATA 2010, there is no more overlap between error bars of the two most precise determinations especially if one considers the revised QED calculations, the new value of the relative electron mass and the latest adjustments of the Rydberg constant and of the rubidium mass, which shifted the value of the fine structure constant $\alpha\left(a_{\mathrm{e}}\right)$ derived from the measurement of electron anomaly 
[29].

Nowadays there is a strong motivation to continue improving the uncertainty on the measurement of the ratios $h / m_{\mathrm{Rb}}$ and $h / m_{\mathrm{Cs}}$. They will improve the reliability of the CODATA value of $h / m_{\mathrm{u}}$, which is sensitive to QED calculations. Furthermore, this will also provide the most precise test of QED to date [33].

\section{Conclusion}

In this paper we discussed the key role of the ratio $h / m_{\mathrm{u}}$ in the foreseen redefinition of the kilogram. A reliable and precise value of this ratio should allow a realization of the kilogram based on the Planck constant by using the XRCD method. We have also presented the state of the art for measurements of fundamental constants and physical quantities that are involved in the determination of this ratio. Nowadays the relative uncertainty on the CODATA 2014 the value of the ratio $h / m_{\mathrm{u}}$ is of $4.5 \times 10^{-10}$, which complies with the recommendations of the Consultative Committee for Mass and Related Quantities (CCM) for the redefinition of the kilogram ([47] and ref herein). However the lack of overlap between the value deduced from the photon recoil measurements and the one determined from $\alpha\left(a_{\mathrm{e}}\right)$ makes the resulting value of ratio $h / m_{\mathrm{u}}$ less reliable. As it appears along the paper, great efforts are being made in many groups around the world to improve the precision of the value of fundamental constants involved in the determination of this ratio.

\section{Acknowledgments}

This experiment is supported by: the Agence Nationale pour la Recherche with FISCOM Project No. ANR-06- BLAN-0192 and INAQED Project No. ANR-12-JS04-0009, IFRAF (Institut Francilien de Recherches sur les Atomes Froids), FIRST-TF Labex, the Direction Générale de l' armement (DGA), and "Emergence" Program of the University Pierre et Marie Curie.

[1] Mills I M, Mohr P J, Quinn T J, Taylor B N and Williams E R 2005 Metrologia 4271 URL http://dx.doi.org/10.1088/0026-1394/42/2/001

[2] Egidi C 1963 Nature 200 61-62 URL http://dx.doi.org/10.1038/200061a0

[3] Bettin H, Fujii K, Man J, Mana G, Massa E and Picard A 2013 Annalen der Physik 525 680-687 ISSN 1521-3889 URL http://dx.doi.org/10.1002/andp. 201300038

[4] Fujii K, Bettin H, Becker P, Massa E, Rienitz O, Pramann A, Nicolaus R, Kuramoto N, Busch I and Borys M 2016 Metrologia Special Issue: Realization, maintenance and dissemination of the new kilogram MET-100701

[5] Kibble B P 1976 A Measurement of the Gyromagnetic Ratio of the Proton by the Strong Field Method (Boston, MA: Springer US) pp 545-551 ISBN 978-1-4684-2682-3 URL http://dx.doi.org/10.1007/978-1-4684-2682-3_80

[6] Steiner R 2013 Reports on Progress in Physics $76 \quad 016101$ URL http://stacks . iop.org/0034-4885/76/i=1/a=016101

[7] Stock M 2013 Metrologia 50 R1 URL http://stacks.iop.org/0026-1394/50/i=1/a=R1 
Precise determination of the ratio $h / m_{\mathrm{u}}$

[8] Robinson I and Schlamminger S 2016 Metrologia Special Issue: Realization, maintenance and dissemination of the new kilogram MET-100705

[9] CGPM 2014 Proceedings of the 25th meeting of the general conference on weights and measures URL http://www.bipm.org/utils/common/pdf/CGPM/CGPM25.pdf

[10] CCM 2014 Report of the 14th meeting of the consultative committee for mass and related quantities URL http://www.bipm.org/utils/common/pdf/CC/CCM/CCM14.pdf

[11] CCM 2014 Draft mise en pratique for the definition of the kilogram URL http://www.bipm.org/en/measurement-units/new-si/\#communication

[12] CCU 2015 Draft of the 9th edition of the si brochure URL http://www.bipm.org/utils/common/pdf/si-brochure-draft-2016.pdf

[13] Audi G, Wang M, Wapstra A, Kondev F, MacCormick M and Xu

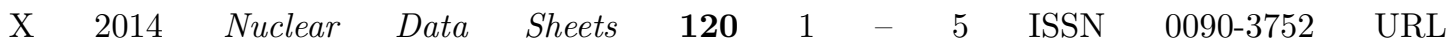
http://www.sciencedirect.com/science/article/pii/S0090375214004438

[14] Bordé C J 2005 Philosophical Transactions of the Royal Society of London A: Mathematical, Physical and Engineering Sciences 363 2177-2201 URL http://rsta.royalsocietypublishing.org/content/363/1834/2177. abstract

[15] Audi G, Wang M, Wapstra A, Kondev F, MacCormick M, Xu X and Pfeiffer B 2012 Chinese Physics $C 361287$ URL http://stacks.iop.org/1674-1137/36/i=12/a=002

[16] Wang M, Audi G, Wapstra A, Kondev F, MacCormick M, Xu X and Pfeiffer B 2012 Chinese Physics C 361603 URL http://stacks.iop.org/1674-1137/36/i=12/a=003

[17] Sturm S, Kohler F, Zatorski J, Wagner A, Harman Z, Werth G, Quint W, Keitel C H and Blaum K 2014 Nature 506 467-470 URL http://dx.doi.org/10.1038/nature13026

[18] Pachucki K, Czarnecki A, Jentschura U D and Yerokhin V A 2005 Phys. Rev. A 72(2) 022108 URL http://link.aps.org/doi/10.1103/PhysRevA.72.022108

[19] Mohr P J, Taylor B N and Newell D B 2012 Rev. Mod. Phys. 84(4) 1527-1605 URL http://link.aps.org/doi/10.1103/RevModPhys.84.1527

[20] Parthey C G, Matveev A, Alnis J, Bernhardt B, Beyer A, Holzwarth R, Maistrou A, Pohl R, Predehl K, Udem T, Wilken T, Kolachevsky N, Abgrall M, Rovera D, Salomon C, Laurent P and Hänsch T W 2011 Phys. Rev. Lett. 107(20) 203001 URL http://link.aps.org/doi/10.1103/PhysRevLett.107.203001

[21] de Beauvoir B, Schwob C, Acef O, Jozefowski L, Hilico L, Nez F, Julien L, Clairon A and Biraben F 2000 Eur. Phys. J. D 12 61-93 URL http://dx.doi.org/10.1007/s100530070043

[22] The nist reference on constants, units, and uncertainty URL http://physics.nist.gov/cuu/Constants/

[23] Pohl R, Antognini A, Nez F, Amaro F D, Biraben F, Cardoso J M R, Covita D S, Dax A, Dhawan S, Fernandes L M P, Giesen A, Graf T, Hänsch T W, Indelicato P, Julien L, Kao C Y, Knowles P, Le Bigot E O, Liu Y W, Lopes J M, Ludhova L, Monteiro C M B, Mulhauser F, Nebel T, Rabinowitz P, dos Santos J M F, Schaller L A, Schuhmann K, Schwob C, Taqqu D, Veloso J F C A and Kottmann F 2010 Nature 466 213-216 URL http://dx.doi.org/10.1038/nature09250

[24] Antognini A, Nez F, Schuhmann K, Amaro F D, Biraben F, Cardoso J M R, Covita D S, Dax A, Dhawan S, Diepold M, Fernandes L M P, Giesen A, Gouvea A L, Graf T, Hänsch T W, Indelicato P, Julien L, Kao C Y, Knowles P, Kottmann F, Le Bigot E O, Liu Y W, Lopes J A M, Ludhova L, Monteiro C M B, Mulhauser F, Nebel T, Rabinowitz P, dos Santos J M F, Schaller L A, Schwob C, Taqqu D, Veloso J F C A, Vogelsang J and Pohl R 2013 Science 339 417-420 ISSN 00368075 (Preprint http://science.sciencemag.org/content/339/6118/417.full.pdf) URL http://science.sciencemag.org/content/339/6118/417

[25] Odom B, Hanneke D, D'Urso B and Gabrielse G 2006 Phys. Rev. Lett. 97(3) 030801 URL http://link.aps.org/doi/10.1103/PhysRevLett.97.030801

[26] Hanneke D, Fogwell S and Gabrielse G 2008 Phys. Rev. Lett. 100(12) 120801 URL http://link.aps.org/doi/10.1103/PhysRevLett.100.120801

[27] Fogwell Hoogerheide S, Dorr J C, Novitski E and Gabrielse 
Precise determination of the ratio $h / m_{\mathrm{u}}$

G 2015 Review of Scientific Instruments $\mathbf{8 6} 053301 \quad$ URL http://scitation.aip.org/content/aip/journal/rsi/86/5/10.1063/1.4919413

[28] Aoyama T, Hayakawa M, Kinoshita T and Nio M 2012 Phys. Rev. Lett. 109(11) 111807 URL http://link.aps.org/doi/10.1103/PhysRevLett.109.111807

[29] Aoyama T, Hayakawa M, Kinoshita T and Nio M 2015 Phys. Rev. D 91(3) 033006 URL http://link.aps.org/doi/10.1103/PhysRevD.91.033006

[30] Yerokhin V A, Berseneva E, Harman Z, Tupitsyn I I and Keitel C H 2016 Phys. Rev. Lett. 116(10) 100801 URL http://link.aps.org/doi/10.1103/PhysRevLett.116.100801

[31] Weiss D S, Young B C and Chu S 1993 Phys. Rev. Lett. 70(18) 2706-2709 URL http://link.aps.org/doi/10.1103/PhysRevLett.70.2706

[32] Bordé C J, Salomon C, Avrillier S, van Lerberghe A, Bréant C, Bassi D and Scoles G 1984 Phys. Rev. A 30(4) 1836-1848 URL http://link.aps.org/doi/10.1103/PhysRevA.30.1836

[33] Bouchendira R, Cladé P, Guellati-Khélifa S, Nez F and Biraben F 2011 Phys. Rev. Lett. 106(8) 080801 URL http://link.aps.org/doi/10.1103/PhysRevLett.106.080801

[34] Estey B, Yu C, Müller H, Kuan P C and Lan S Y 2015 Phys. Rev. Lett. 115(8) 083002 URL http://link.aps.org/doi/10.1103/PhysRevLett.115.083002

[35] Wicht A, Hensley J M, Sarajlic E and Chu S 2002 Physica Scripta 200282 URL http://stacks.iop.org/1402-4896/2002/i=T102/a=014

[36] Ben Dahan M, Peik E, Reichel J, Castin Y and Salomon C 1996 Phys. Rev. Lett. 76(24) 4508-4511 URL http://link.aps.org/doi/10.1103/PhysRevLett.76.4508

[37] Wilkinson S R, Bharucha C F, Madison K W, Niu Q and Raizen M G 1996 Phys. Rev. Lett. 76(24) 4512-4515 URL http://link.aps.org/doi/10.1103/PhysRevLett.76.4512

[38] Cladé P 2015 Rivista Del Nuovo Cimento 38 173-207

[39] Bouchendira R, Cladé P, Guellati-Khélifa S, Nez F and Biraben F 2013 Annalen der Physik 525 484-492 ISSN 1521-3889 URL http://dx.doi.org/10.1002/andp. 201300044

[40] Andia M, Étienne Wodey, Biraben F, Cladé P and Guellati-Khélifa S 2015 J. Opt. Soc. Am. B 32 1038-1042 URL http://josab.osa.org/abstract.cfm?URI=josab-32-6-1038

[41] Cladé P, Guellati-Khélifa S, Nez F and Biraben F 2009 Phys. Rev. Lett. 102(24) 240402 URL http://link.aps.org/doi/10.1103/PhysRevLett.102.240402

[42] Cladé P, Plisson T, Guellati-Khélifa S, Nez F and Biraben F 2010 The European Physical Journal D 59 349-360 ISSN 1434-6079 URL http://dx.doi.org/10.1140/epjd/e2010-00198-0

[43] Jannin R, Cladé $\mathrm{P}$ and Guellati-Khélifa $\mathrm{S} 2015$ Phys. Rev. A 92(1) 013616 URL http://link.aps.org/doi/10.1103/PhysRevA.92.013616

[44] Müller H, Chiow S w, Herrmann S and Chu S 2009 Phys. Rev. Lett. 102(24) 240403 URL http://link.aps.org/doi/10.1103/PhysRevLett.102.240403

[45] Lan S Y, Kuan P C, Estey B, English D, Brown J M, Hohensee M A and Müller H 2013 Science $\mathbf{3 3 9} \quad 554-557 \quad$ ISSN $0036-8075$ (Preprint http://science.sciencemag.org/content/339/6119/554.full.pdf) URL http://science.sciencemag.org/content/339/6119/554

[46] Mohr P J, Newell D B and Taylor B N 2015 arXiv:1507.07956 [physics.atom-ph] URL http://arxiv.org/abs/1507.07956

[47] Pinot P, Beaudoux F, Bentouati D, Espel P, Madec T, Thomas M, Silvestri Z, Ziane D and Piquemal F 2016 Metrologia 531139 URL http://stacks.iop.org/0026-1394/53/i=4/a=1139 